\title{
Germanica
}

\section{Der Mensch im Spiegel von 'Beerenaugen'. Yoko Tawadas Roman Etüden im Schnee (2014)}

\section{Lina Werry}

\section{Q OpenEdition}

\section{$\checkmark$ Journals}

Édition électronique

URL : http://journals.openedition.org/germanica/3891

DOI : 10.4000/germanica.3891

ISSN : 2107-0784

Éditeur

Université de Lille

\section{Édition imprimée}

Date de publication : 30 juin 2017

Pagination : 215-221

ISBN : 9782913857391

ISSN : 0984-2632

\section{Référence électronique}

Lina Werry , „Der Mensch im Spiegel von 'Beerenaugen'. Yoko Tawadas Roman Etüden im Schnee (2014)", Germanica [Online], 60 | 2017, Online erschienen am: 30 Juni 2017, abgerufen am 07 Januar 2021. URL: http://journals.openedition.org/germanica/3891 ; DOl: https://doi.org/10.4000/germanica. 3891

\section{(C) Tous droits réservés}




\title{
(Re)lectures \\ Der Mensch im Spiegel von 'Beerenaugen'. Yoko Tawadas Roman Etüden im Schnee (2014)
}

\author{
Lina WERRY \\ Lille
}

Die Figur des Tierpflegers Matthias erwartet sich von seinem Zusammentreffen mit dem Eisbärjungen Knut die angebliche schockierende Leere in den Bärenaugen, von der Forscher berichten, wiederzufinden. Stattdessen blickt er in Augen, die ihn selbst mit all seinen menschlichen Makeln widerspiegeln. Angesichts der ,gereiften, saftigschwarzen Beerenaugen“ (S. 243) erstarrt selbst der Blick des Fotografen, der seine Kameralinse auf den kleinen Bären richtet. Wer ist hier der Überlegene?

Etüden im Schnee ist Yoko Tawadas jüngster Roman, 2014 im konkursbuch Verlag erschienen ${ }^{1}$. Sie wählt darin als Erzählinstanzen Eisbären, was auf den ersten Blick verwundern mag. Doch ein Verständnis ihrer Werke erfordert immer einen zweiten und sogar dritten Blick und so versinnbildlicht die Rekurrenz auf Eisbären in ihrem aktuellen Buch die vielleicht wichtigste Intention der Autorin: die Durchbrechung von Lesegewohnheiten, um ,,versteinerte Köpfe“ gar nicht erst entstehen zu lassen. Es sei wichtig, ,dass der Geist immer wieder gedehnt und gedehnt wird“, zitiert sie eine Nebenfigur in Etüden

1. - Yoko Tawada, Etüden im Schnee. Roman, Tübingen, konkursbuch Verlag, 2014.

GERMANICA, 2017, LX, pp. 215 à 221. 
im Schnee (S. 295). Das natürliche Habitat von Eisbären erstreckt sich auf die nördlichen Teile der drei Kontinente Nordamerika, Asien und Europa. Insofern ist der Roman auch zu verstehen als eine Abkehr von eindimensionalen (oft eurozentristischen) Perspektiven.

Da in Tawadas Texten gewöhnlich mehrere Diskurse zusammenlaufen, drängen sich intertextuelle Vergleiche zwischen ihren Werken auf. So berührt Etüden im Schnee stellvertretend für andere Werke Fragen nach dem Verhältnis von Sprache und Fremdsprache, der Geschlechter, von Identität und Heimat, Realität und Traum, Erinnerung und Erdichtung. Hinzukommen, themenbedingt, das Verhältnis zwischen Mensch und Tier sowie eine kritische Reflexion der Hoheitsstellung, die sich der Mensch darin zuschreibt.

Konkrete intertextuelle Verweise auf Franz Kafka und Heinrich Heine stellen den Roman in eine Traditionslinie mit Autoren von phantastischer Tierliteratur. So erwirbt die namenlose Eisbärin im ersten Kapitel in einer Buchhandlung Heinrich Heines Versepos Atta Troll, nachdem sie wie durch Zufall eine illustrierte Seite aufgeschlagen hatte: „und dort lag ein ausgestreckter Bär mit ausgestreckten Vorder- und Hinterbeinen.“ (S. 70) Atta Troll weist viele Motive auf, die in Etüden im Schnee weiterverarbeitet werden, etwa der gewaltsame Missbrauch von Bären als Tanzbären, die sprachliche Verständigung zwischen Mensch und Tier, der Eintritt in die Traumwelt, die Widerlegung der Menschenrechte als ,natürlich', die Infragestellung der selbstetablierten menschlichen Überlegenheit. Hingegen wird Atta Troll, anders als die Bären bei Tawada, als negative Figur gezeichnet, deren politische Überzeugungen letztlich dekonstruiert werden².

Franz Kafkas Erzählung Forschungen eines Hundes inspiriert die Bärin, über das Schreiben zu sinnieren: „Warum darf ich nicht eine Gegenwart schreiben? Warum muss ich eine Vergangenheit erfinden, die wahrhaftig klingt?" (S. 81) Bereits hier wird die Möglichkeit angedeutet, dass die Geschichten der Kapitel zwei und drei aus der Feder der Bärin stammen.

Auch auf die durchgehende Komik des Romans sei verwiesen: Indem aus der Sicht von Eisbären erzählt wird, entsteht ein Spiegelungseffekt, durch den die Menschen mit ihrem eigenen Verhalten konfrontiert werden und - hier entsteht der komische Effekt - es in ihrer Ignoranz selbst noch nicht einmal merken.

Etüden im Schnee umfasst 312 Seiten, die in drei annähernd gleichstarke Kapitel untergliedert sind, welche inhaltlich drei Eisbärengenerationen entsprechen: Evolutionstheorie der Großmutter, Der Todeskuss und Im Andenken an den Nordpol. Nähert man sich dem

2. - - Vgl. Heinrich Heine, Atta Troll. Ein Sommernachtstraum, Hamburg, Hoffmann und Campe, 1868. 
Buch zunächst auf paratextueller Ebene, fällt auf, dass bereits durch das Element der graphischen Gestaltung - Bilder von Eisblumen vor blauem Hintergrund, eine Nahaufnahme von Eisbärfell, glitzernde Eiskristalle -, die die Kapitel optisch voneinander abgrenzen, eine Atmosphäre erzeugt wird, die die Leser in die Stimmung des Romans einführt. Auch der Buchumschlag präsentiert Ausschnitte von Fotografien einer Eisbärenfamilie. Mehrere Sinne werden angesprochen, die Überschreitung der reinen Ebene der Kognition wird bereits formal angedeutet. Die Fotografien stehen im Kontrast zu dem Inhalt, da die tierischen Figuren hier von der Arktis nur träumen können. Sie sind in Gefangenschaft aufgewachsen und dazu gezwungen, sich an eine für sie unnatürliche menschliche Welt anzupassen.

Der Romanplot ist inspiriert von realen Vorlagen. Dies wird besonders im letzten Kapitel deutlich, in dem von Knut die Rede ist, einem Eisbären, der 2006 im Berliner Zoologischen Garten geboren und durch die Medien zu einem Star stilisiert und zu einer vielverkauften Marke wurde. Von seinen Eltern, Toska und Lars, wird im zweiten Kapitel erzählt, insbesondere von Toska, die in Kanada geboren, im Staatszirkus der DDR dressiert und dann an den Berliner Zoo verkauft wurde. Auch die Bärendresseurin Barbara beruht auf einer realen Vorlage - stellt der Name Barbara eine lautliche Reminiszenz an ,Bär ${ }^{\star}$ dar, ist dies nicht minder der Fall bei der realen Person Ursula Böttcher, berühmteste Dompteurin der DDR, die mit der Bärin Toska den berüchtigten ,Todeskuss' vorgeführt hat, der im zweiten Kapitel des Romans eine wichtige Rolle spielt. Ihre wahre Biographie entspricht in weiten Teilen der der fiktiven Figur Barbara. Auch die Figur des Tierpflegers Matthias im dritten Kapitel ist an die reale Person Thomas Dörflein angelehnt, die durch die Aufzucht von Eisbär Knut Berühmtheit erlangt hat.

Drei Geschichten werden erzählt, die durch die Verwandtschaft der tierischen Figuren miteinander verknüpft sind. Eine weitere Gemeinsamkeit der Eisbären ist, dass sie alle in Gefangenschaft und von Menschen aufgezogen wurden. Die Bärinnen in den ersten beiden Kapiteln sind dressierte Zirkustiere, während ihr Nachkomme Knut, Hauptfigur des dritten Kapitels, in einem Zoo lebt. Die Bären haben alle menschliche Attribute, ohne den Menschen gleich zu sein, was ihnen einen distanziert-kritischen Blick auf den Homo sapiens bewahrt.

Im ersten Kapitel Evolutionstheorie der Großmutter, das zur Zeit der DDR in Moskau spielt, ist die Hauptfigur eine Eisbärin, die von Tierpfleger Iwan aufgezogen und $\mathrm{zu}$ einem Zirkusbären dressiert wurde. Aufgrund eines Hüftschadens kann sie nicht mehr im Zirkus auftreten und nimmt fortan an politischen Konferenzen zu Themen wie Der Stolz der Arbeiterklasse teil. Um ihre schmerzhafte Kindheit, die durch ihre Dressur geprägt ist, zu verarbeiten, beginnt sie, Bücher zu 
schreiben. Ein Verleger, Seelöwe, dessen Avancen sie als Zirkusstar ausschlug, veröffentlicht ihre Autobiographie unter dem pathetischen Titel Applaussturm für meine Tränen, die sie auch international zum Star macht. Sie wird - vorgeblich zur Teilnahme an einer Konferenz von einer Bürgerinitiative nach Westberlin eingeladen, wo sie bleiben wird. Doch die Fülle an Angeboten im kapitalistischen Westen hindern die Autorin am Schreiben und lassen sie ihre alte Heimat vermissen: „Mein Traum war, in einer schneidenden Kälte eine neue Nummer für die Bühne einzuüben um den Donnerwetterbeifall des Publikums zu kassieren." (S. 101) Schließlich entscheidet sie sich, nach Kanada zu emigrieren, in ein Land, das ihr in erster Linie Kälte verspricht. Während sie in Büchern über Kanada stöbert, beginnen sich die Ebenen Realität und Traum zu überlagern. So liest sie eine Liebesgeschichte, in die sie sich hineinprojiziert. Sie lernt ihren Mann Christian kennen, mit dem sie „wie auf Knopfdruck“ (S. 91) ihre Tochter Toska bekommt - im nächsten Abschnitt heißt es: „Während ich diese Passagen aus dem Buch abschrieb, stieg ich als eine Protagonistin in die erzählte Geschichte hinein.“ (S. 91) Dies stellt einen Vorausgriff auf das folgende zweite Kapitel dar, das das Leben von Toska behandeln wird. Auch auf das dritte Kapitel und die Geburt ihres Enkels Knut wird bereits verwiesen, was die Deutung plausibel macht, dass es sich bei den beiden folgenden Geschichten um eine Fiktion der Bärin handelt: „Sie (Toska) wird später einen Sohn gebären, der so liebenswürdig aussieht, dass man ihn sofort knuddeln möchte. Ich werde ihn, mein erstes Enkelkind, ,Knut " nennen.“ (S. 93) Auf Wunsch ihres dänischen Mannes verlassen sie nach Toskas Geburt Kanada und gehen zurïck in die DDR.

Das zweite Kapitel, Todeskuss, wird aus der Ich-Perspektive von Barbara erzählt, Bärendresseurin in einem Zirkus in der DDR. Auf die phonetische Nähe des Namens ,Barbara' zu ,Bär' verweist der Text selbst: „Bar wie Bär.“ (S. 164) - auf Latein: ,Urs‘, was die Verbindung zur realen Person Ursula Böttcher herstellt. Die Bärin Toska, Schauspielerin in einem Kindertheater, kommt durch eine Initiative Barbaras zum Zirkus. Es entwickelt sich ein Vertrauensverhältnis zwischen Dresseurin und Dressierter, was so weit führt, dass beide anfangen, in einer gemeinsamen Sprache miteinander zu kommunizieren: „Ich saß auf derselben Eisscholle wie Toska und verstand jedes Wort, das sie mir sagte." (S. 123) Wie angedeutet wird, ist die gemeinsame Sprache der beiden auf die Sphäre des Traumes beschränkt, genauer begegnen sie sich in einer „Sphäre, die sich zwischen der der Tiere und der Menschen befand.“ (S. 129) Der Verweis auf den ,ritten Raum' stellt eine Reminiszenz an transkulturelle Theorien, genauer an Homi K. Bhabas Theorie des ,third space', dar und zielt somit auf eine Auflösung der traditionellen Hierarchie zwischen Mensch und Tier. In diesem dritten Raum entwickelt sich Toska zu einer Art Alter Ego für Barbara, der sie ihre 
innersten Ängste anvertraut. Je mehr sie sich der Bärin gegenüber öffnet, desto mehr verschließt sie sich vor ihrem Ehemann Markus, ebenfalls Bärendresseur am Zirkus. Dieser verrennt sich in seiner Eifersucht und der Idee, seine Frau sei psychisch erkrankt. Sein Tod durch einen Kodiakbären - der ebenfalls der Realität entspricht - wird im Roman in einem Atemzug mit dem Fall der Berliner Mauer genannt. Lakonisch heißt es: „Die DDR starb, und so auch mein Mann.“ (S. 182)

Interessant ist ein weiteres Mal der Bezug zur Schriftstellerei: Toska selbst sagt, sie könne nicht schreiben, da sie bereits als Figur in der Autobiographie ihrer Mutter vorkomme. Aus diesem Grund beginnt Barbara, Toskas Biographie aufzuschreiben. Um sich in dieser Aufgabe zu üben, erzählt sie ihre eigene Lebensgeschichte, ihre Kindheit und Jugend, ihre Liebe zum Zirkus und ihren schwierigen Eintritt als Außenstehende in dessen feste Gemeinschaft. Aus der geplanten Biographie wird eine Autobiographie.

Markiert durch einen graphischen Tatzenabdruck, wird gegen Ende des Kapitels ein Perspektivwechsel hin zu Toska eingeleitet. Aus der Sicht Toskas wird von dem Höhepunkt ihrer Aufführung, dem „Todeskuss“ berichtet. Dabei nimmt die Bärin mit ihrer Zunge ein Stück Zucker aus Barbaras Mund. Barbaras Seele, so Toska, gehe bei jedem Kuss in ihren Bärenkörper über. Für sie sei die menschliche Seele jedoch weit weniger romantisch als angenommen; sie bestehe hauptsächlich aus ,vielen kaputten Sprachscherben, den Schatten der Sprachen und den Bildern, die nicht Wörter werden konnten.“ (S. 203) Diese wenig schmeichelhafte Beschreibung der menschlichen Seele als Altteillager für Sprachreste ist als eine ironische Kritik an der Überhöhung der Bedeutung der menschlichen im Vergleich zur tierischen Seele zu lesen.

Die Reflexion über Sprache ist wiederum das verbindende Element aller Werke Tawadas. Eine ihrer Grundannahmen besteht darin, dass jede Sprache fremd und dem Menschen nicht durch Geburt gegeben sei. So sei die Bezeichnung ,Muttersprache“ irreführend, da diese nicht einfach mit der Muttermilch aufgesogen würde, sondern, wie jede andere Sprache auch, gelernt werden müsse: „Was ist meine Muttersprache?“, fragt Toska und erhält die schlichte Antwort: „Die Sprache deiner Mutter.“ (S. 67), die sie verwirrt zurücklässt, da sie nie mit ihrer Mutter gesprochen hat. Während die sogenannte ,Muttersprache' Gedanken fest an Bedeutungen hefte (was Tawada mit dem Bild der Heftklammer veranschaulicht), löse eine Fremdsprache diese Bindung zwischen Wort und Bedeutung auf (sie sei ein „Heftklammerentferner“3). In Etüden im Schnee ist eine sprachliche Verständigung zwischen Tier und Mensch erst durch das Medium des Traumes möglich. Gesprochen wird dann

3. - Yoko Tawada, „Von der Muttersprache zur Sprachmutter. Eine Begegnung mit dem Deutschen“, NZZ Folio, Oktober 1994. 
allerdings über essentiellere Dinge als in der sogenannten Realität. Lässt sich eine Grenze zwischen den beiden Sphären dann noch so ohne weiteres ziehen? Wird die sogenannte Realität nicht zur Fiktion, wenn in ihr nur Belangloses verhandelt wird? Zumindest wird die Möglichkeit dazu aufgezeigt.

Dadurch, dass Barbaras Seele in Toska geflossen sei, ist sie imstande, die Biographie Barbaras aufzuschreiben, die sich nach der Auflösung des Zirkus enttäuscht von der Welt zurückgezogen hat. Die Schriftstellerei wird, ähnlich wie bei ihrer Mutter, zu ihrer Hauptbeschäftigung. Sie ist wichtiger als ihr bald darauf geborener Sohn Knut, der die dritte Bärengeneration verkörpert und die Hauptfigur des dritten und letzten Kapitels darstellt.

Mit dem dritten Kapitel Im Andenken an den Nordpol ist die Geschichte im 21. Jahrhundert angekommen. Der kleine Eisbär Knut wird von Tierpfleger Matthias mit der Flasche aufgezogen und erlangt - wie der reale Knut - Berühmtheit durch die Medien. Matthias ist ein introvertierter Einzelgänger, der Journalisten scheut. Nur Knut gegenüber öffnet er sich - worin eine Parallele zu Barbara erkennbar wird, die ihr wahres Ich auch nur vor Toska offenbaren konnte. Mit seiner Gitarre weiht er Knut in die Welt der Musik ein, was auf den mehrdeutigen Titel des Romans, Etüden im Schnee, verweist. Indem Knut auf die Geräusche - Musik in seinen Ohren - von draußen achtet, gelingt es ihm, temporär aus seinem Käfig zu fliehen: „Die gehörte Welt war so geräumig und farbenreich, dass die gesehene Welt sie nicht übertreffen könnte.“ (S. 224)

Als Knut alt genug ist, darf er seinen Käfig verlassen und mit Matthias durch den Zoo spazieren. Er vergleicht diese Spaziergänge mit einer „akademischen Ausbildung“ (S. 261), bei der er Neues über sich und seine Umwelt erfährt. Dahingegen seien die ,Shows', bei denen er durch einfache Kunststücke Menschenmassen begeistert, eine Fingerübung, eine Etüde. „Es ist manchmal sehr leicht, den ausgewachsenen Homo sapiens glücklich zu machen, weil er von einer kindlichen Natur ist.“ (S. 264)

Hatten sich seine Mutter und Großmutter der Schriftstellerei zugewandt, bleibt dieses Talent Knut verwehrt. Es wird so viel über ihn geschrieben, dass für seine Geschichten kein Raum bleibt. Er selbst äußert eine Medienkritik: „Man nahm mir ein Stück Leben weg und sperrte es im Zeitungspapier ein.“ (S. 276) Aus einem Zeitungsartikel erfährt Knut schließlich auch vom Tod Matthias'; auch dessen reale Vorlage, der Tierpfleger Thomas Dörflein, starb zwei Jahre nach Knuts Geburt an einem Herzinfarkt.

Eine neue, rätselhafte Figur tritt spät im Kapitel auf: Michael. Stellt man die Verbindung zu der russischen Koseform Mischa her, so stellt sich heraus, dass diese ein Synonym für ,Bär" ist. Michael repräsentiert 
Knuts Wandel von einem süßen Teddy hin zu einem ausgewachsenen Bären, der während einer Aufführung vor den Augen des entsetzten Publikums seinen Pfleger Matthias unabsichtlich verletzt hat. Er selbst wurde als Kind gefeiert und als Jugendlicher fallengelassen. Michael erscheint als Scharnierfigur zwischen Aufstieg und Fall und könnte somit als Todesbote für Knut fungieren. Der reale Knut starb an einer Autoimmunerkrankung, einer Menschenkrankheit, wie Forscher inzwischen herausgefunden haben. Tawada konnte davon nichts wissen - die Forschungsergebnisse erschienen erst nach der Veröffentlichung ihres Romans - doch würde sich die Todesursache perfekt in die Logik des Romans einreihen: Ein gefangener Bär, der gezwungen wird, Menschen zu unterhalten, stirbt letztlich an seiner vermeintlichen Menschwerdung. Der Roman hingegen endet, traurig und hoffnungsvoll zugleich, mit der Erfüllung von Knuts sehnlichstem Wunsch: „Es schneit!“ (S. 312)

Die Vielzahl an Geschichten im Roman, erzählt von unterschiedlichen Stimmen, die allesamt als ,Etüden', also Studien für die Gesamterzählung gelten können, macht das Leseereignis selbst zu einer nie enden wollenden Etüde. Dieser bedarf es, um in die Tiefenschichten des Romans vordringen zu können. Die Schönheit der sprachlichen Musik des Romans bleibt dabei ungebrochen.

Yoko Tawada, 1960 in Tokyo geboren, ist Schriftstellerin und promovierte Literaturwissenschaftlerin mit Wohnsitz in Berlin. Ihre literarischen Werke, die alle Gattungen umfassen, veröffentlicht sie auf Deutsch und Japanisch. Für ihre Arbeiten erhielt sie zahlreiche Auszeichnungen, zuletzt 2016 den Kleist-Preis. Sie ist Mitglied der Deutschen Akademie für Sprache und Dichtung sowie der Mainzer Akademie der Wissenschaften und der Literatur und Gastdozentin an wechselnden internationalen Hochschulen. 
